\title{
Vitamin D metabolism-related genetic variants, dietary protein intake and improvement of insulin resistance in a 2 year weight-loss trial: POUNDS Lost
}

\author{
Qibin $\mathrm{Qi}^{1,2}$ - Yan Zheng ${ }^{2}$ - Tao Huang ${ }^{2}$. Jennifer Rood ${ }^{3}$ - George A. Bray ${ }^{3}$. \\ Frank M. Sacks ${ }^{2} \cdot \mathrm{Lu} \mathrm{Qi}^{2,4,5}$
}

Received: 8 May 2015 / Accepted: 21 August 2015 /Published online: 29 September 2015

(C) Springer-Verlag Berlin Heidelberg 2015

\begin{abstract}
Aims/hypothesis Vitamin D and related genetic variants are associated with obesity and insulin resistance. We aimed to examine whether vitamin D metabolism-related variants affect changes in body weight and insulin resistance in response to weight-loss diets varying in macronutrient content.

Methods Three vitamin D metabolism-related variants, $D H C R 7$ rs12785878, CYP2R1 rs10741657 and $G C$ rs2282679, were genotyped in 732 overweight/obese participants from a 2 year weight-loss trial (POUNDS Lost). We assessed genotype effects on changes in body weight, fasting levels of glucose and insulin, and HOMA-IR at 6 months (up to 656 participants) and 2 years (up to 596 participants) in response to low-protein vs highprotein diets, and low-fat vs high-fat diets.

Results We found significant interactions between $D H C R 7$ rs 12785878 and diets varying in protein, but not in fat, on
\end{abstract}

Qibin Qi and Yan Zheng contributed equally to this work.

Electronic supplementary material The online version of this article (doi:10.1007/s00125-015-3750-1) contains peer-reviewed but unedited supplementary material, which is available to authorised users.

\section{Lu Qi}

lqi1@tulane.edu

1 Department of Epidemiology and Population Health, Albert Einstein College of Medicine, Bronx, NY, USA

2 Department of Nutrition, Harvard T.H. Chan School of Public Health, 665 Huntington Avenue, Boston, MA 02115, USA

3 LSU, Pennington Biomedical Research Center, Baton Rouge, LA, USA

4 Department of Epidemiology, School of Public Health and Tropical Medicine, Tulane University, New Orleans, LA 70112, USA

5 Channing Laboratory, Brigham and Women's Hospital and Harvard Medical School, Boston, MA, USA changes in insulin and HOMA-IR at both 6 months ( $p$ for interaction $<0.001$ ) and 2 years ( $p$ for interaction $\leq 0.03$ ). The T allele (vitamin-D-increasing allele) of DHCR7 rs12785878 was associated with greater decreases in insulin and HOMA-IR $(p<0.002)$ in response to high-protein diets, while there was no significant genotype effect on changes in these traits in the low-protein diet group. Generalised estimating equation analyses indicated significant genotype effects on trajectory of changes in insulin resistance over the 2 year intervention in response to high-protein diets $(p<0.001)$. We did not observe significant interaction between the other two variants and dietary protein or fat on changes in these traits. Conclusions/interpretation Our data suggest that individuals carrying the T allele of DHCR 7 rs12785878 might benefit more in improvement of insulin resistance than noncarriers by consuming high-protein weight-loss diets.

Trial registration: ClinicalTrials.gov NCT00072995

Keywords Diet intervention · Genetic variants · Insulin resistance $\cdot$ Vitamin D $\cdot$ Weight loss

Abbreviations
$\begin{array}{ll}\text { DHCR7 } & \text { 7-Dehydrocholesterol reductase } \\ \text { GWAS } & \text { Genome-wide association study } \\ \text { 25(OH)D } & \text { 25-Hydroxyvitamin D } \\ \text { POUNDS } & \text { Preventing Overweight Using Novel Dietary } \\ \text { Lost } & \text { Strategies Trial } \\ \text { SNP } & \text { Single nucleotide polymorphism }\end{array}$

\section{Introduction}

Vitamin D has a pleiotropic role in various metabolic functions beyond regulation of calcium and bone metabolism [1]. 
Compelling evidence has shown that low blood levels of 25-hydroxyvitamin D (25(OH)D), an indicator of circulating vitamin D levels, are associated with obesity, insulin resistance and an increased risk of type 2 diabetes [2-8]. A recent genome-wide association study (GWAS) identified that variants in several genes involved in vitamin D metabolism, such as DHCR7, CYP2R1 and GC, were associated with $25(\mathrm{OH}) \mathrm{D}$ levels [9]. The DHCR7 gene encodes 7-dehydrocholesterol reductase (DHCR7), which converts 7-dehydrocholesterol (a precursor both for vitamin D and for cholesterol) to cholesterol, thereby reducing the availability of this precursor for cutaneous synthesis into vitamin $D$ by the action of sunlight [10]. The enzyme encoded by CYP2R1, cytochrome P450, family 2 , subfamily $R$, polypeptide 1 , is a key vitamin $D$ 25 -hydroxylase [11]. The $G C$ gene encodes vitamin $\mathrm{D}$ binding protein, which plays a major role in vitamin $\mathrm{D}$ transport and storage [12]. However, the causal relationships between vitamin D levels and cardiometabolic diseases inferred by Mendelian randomisation studies remain unclear [4, 13-15]. For example, a vitamin $D$ genetic score (based on variants in DHCR7 and CYP2R1) was found to be associated with blood pressure and hypertension [13], but this score was not associated with obesity in a previous analysis [4]. Afzal et al [14] reported that genetic variants associated with lower plasma $25(\mathrm{OH}) \mathrm{D}$ concentrations are associated with an increased risk of type 2 diabetes, while another study did not observe such associations [15]. Thus, examining whether vitamin D metabolism-related genetic variants affect long-term changes in body weight, glucose metabolism and insulin resistance may help explain the role of vitamin $\mathrm{D}$ in cardiometabolic diseases.

It has been shown that DHCR7 activity and DHCR7 gene expression could be regulated by vitamin $\mathrm{D}$ or other dietary nutrients [16-19], suggesting potential interactions between dietary factors and vitamin D metabolism-related genes. Protein-rich and fat-rich foods are the main sources of naturally occurring cholecalciferol (vitamin $\mathrm{D}_{3}$ ) [20]; therefore, we hypothesised that diets varying in protein or fat content might differentially affect the effect of vitamin D metabolism-related variants on body weight and related metabolic traits. Thus, we tested this hypothesis among 732 participants in a 2 year randomised dietary intervention weight-loss trial, the Preventing Overweight Using Novel Dietary Strategies Trial (POUNDS Lost) [21]. We examined the effects of three GWAS-identified vitamin D metabolism-related variants in the DHCR7, CYP2R1 and GC genes on weight loss and improvement of glucose and insulin metabolism in response to diets varying in protein or fat content.

\section{Methods}

Study participants POUNDS Lost is a randomised intervention trial in which 811 overweight or obese participants aged
30-70 years were assigned to one of four energy-limited diets over a 2 year period, in order to compare the effects of energyreduced diets with different compositions of fat, protein and carbohydrate on weight loss. The detailed study design and methods have been described before [21]. Participants were excluded from this trial if they had diabetes treated with medication or unstable cardiovascular disease, if they used medications influencing body weight or if they expressed insufficient motivation at baseline examination. According to the two-by-two factorial design, two diets were low fat ( $20 \%$ of energy derived from fat) and two were high fat ( $40 \%$ of energy derived from fat), and two were average protein $(15 \%$ of energy derived from protein) and two were high protein ( $25 \%$ of energy derived from protein). All these diets consisted of similar foods and met guidelines for cardiovascular health. The caloric prescription for each participant represented a deficit of $3,138 \mathrm{~kJ}(750 \mathrm{kcal}) /$ day from baseline, as calculated from the participant's resting energy expenditure and activity level.

After 2 years, $80 \%$ of the participant $(n=645)$ completed the trial. The main results of weight loss in POUNDS Lost have been reported before [21]. Most of the weight loss occurred in the first 6 months, and the amount of weight loss after 2 years was similar among different diet groups. Both self-reported dietary data and biomarkers confirmed that participants modified their intake of macronutrients in the direction of the assigned goals [21]. In the current study, a total of 732 participants with genotype data available were included (90\% of the participants in POUNDS Lost). At 6 months, 656 participants with body weight measurement and 626 participants with blood samples were included for the analyses. At 2 years, 596 participants with body weight measurements and 533 participants with blood samples were included for the analyses.

The study was approved by the human participants committee at the Harvard T.H. Chan School of Public Health and Brigham and Women's Hospital, Boston, MA, and the Pennington Biomedical Research Center, Baton Rouge, LA, and by a data and safety monitoring board appointed by the National Heart, Lung, and Blood Institute. Written informed consent was provided by all participants.

Measurements At baseline examination and at 6 month and 2 year visits, body weight for each participant was measured in the morning before breakfast. Height was measured at baseline only. BMI was presented as weight $/ h_{\text {eight }}{ }^{2}\left(\mathrm{~kg} / \mathrm{m}^{2}\right)$. In the current study, ethnicity was self-reported and grouped as white, black and others. Fasting blood samples were collected at baseline and at 6 month and 2 year visits. Fasting glucose and insulin were measured using an immunoassay with chemiluminescent detection on the Immulite Analyzer (Diagnostic Products Corporation, Los Angeles, CA, USA). Analyses of glucose and insulin were performed at the 
Clinical Laboratory at Pennington Biomedical Research Center. Insulin resistance was estimated by HOMA-IR [22]: fasting glucose $(\mathrm{mmol} / \mathrm{l}) \times$ fasting insulin $(\mathrm{pmol} / 1) / 156.3$. Dietary intake was assessed by a review of the 5 day diet records at baseline and by $24 \mathrm{~h}$ recall during a telephone interview on three nonconsecutive days at 6 months and at 2 years [21].

Genotyping DNA was extracted from the buffy coat fraction of centrifuged blood using the QIAmp Blood Kit (Qiagen, Chatsworth, CA, USA). Three lead single nucleotide polymorphisms (SNPs) in vitamin-D-related loci, DHCR7 rs12785878 $\left(p=2.1 \times 10^{-27}\right), C Y P 2 R 1$ rs $10741657\left(p=3.3 \times 10^{-20}\right)$ and GC rs2282679 $\left(p=1.9 \times 10^{-109}\right)$, identified through a previous large-scale GWAS for 25-hydroxyvitamin D concentrations (up to 33,996 individuals) [9], were selected in the current study. Genotyping was performed using the OpenArray SNP Genotyping System (BioTrove, Woburn, MA, USA). The genotype success rate was 99\%. Replicate quality control samples $(10 \%)$ were included and genotyped with $>99 \%$ concordance. The genotype frequencies in all participants or in white participants were in Hardy-Weinberg equilibrium $(p>0.05)$.

Statistical analysis General linear models for continuous variables and $\chi^{2}$ tests for categorical variables were applied for the comparison according to genotype groups at baseline. To improve normality, fasting insulin and HOMA-IR were $\log _{10}$ transformed before analyses. The primary outcomes were changes in body weight, fasting insulin, glucose and HOMA-IR over the time the participants remained in the trial. General linear models were applied to compare changes from baseline in these outcomes across genotype groups according to diet groups (high-protein vs low-protein diets and high-fat vs low-fat diets) at 6 month and 2 year visits, adjusted for age, sex, ethnicity and baseline value for the respective outcome. We used additive inheritance models (e.g. DHCR7 rs12785878 genotype groups were coded as 0,1 and 2 in continuous form for GG, GT and TT) in the analyses. To test potential gene-diet intervention interactions, a genotype-by-diet interaction term (e.g. DHCR7 rs12785878 genotype $\times$ high-/low-protein diet group) was included in the models. In addition, generalised estimating equation methods were applied to test genotype effects on the trajectory of changes in fasting insulin and HOMA-IR over the 2 year intervention. As HOMA-IR was calculated on the basis of glucose and insulin, and the endpoints at 6 months and 2 years were correlated, we did not treat them as independent variables. All statistical analyses were performed in SAS version 9.4 (SAS Institute, Cary, NC, USA).

Bonferroni's adjustment was applied to adjust $p$ values for 18 independent tests ( 3 independently measured traits [body weight, glucose and insulin $] \times 3$ genetic variants $\times 2$ diet comparisons) at each time point $(p<0.0028)$. We used Quanto 1.2.4 (University of Southern California, Los Angeles, CA, USA;
http://biostats.usc.edu/Quanto.html) to estimate the detectable effect sizes of genotype-by-diet interactions. For the DHCR7 rs 12785878 variant, the study had $80 \%$ power to detect genediet interaction effect sizes of 2.55 and $3.53 \mathrm{~kg}$ for weight loss, 0.22 and $0.25 \mathrm{mmol} / 1$ for changes in fasting glucose levels, 0.22 and $0.24 \log$-transformed unit for changes in fasting insulin levels, and 0.24 and 0.26 log-transformed unit for changes in HOMA-IR at 6 months and 2 years, respectively, at a $p<0.0028$ level. More power calculation results are shown in electronic supplementary material (ESM) Table 1.

\section{Results}

Baseline characteristics Table 1 shows the baseline characteristics of participants according to the genotypes of $D H C R 7$ rs12785878, CYP2R1 rs10741657 and $G C$ rs2282679. For all three genetic variants, the genotype frequencies were similar between men and women, high- and low-protein diet groups and high- and low-fat diet groups, while they differed among ethnic groups $(p \leq 0.05)$. In addition, the CYP2R1 rs10741657 variant was associated with fasting glucose levels at baseline.

Weight loss and dietary intake Consistent with the entire POUNDS Lost [21], the amount of weight loss was similar between the low- and high-protein diet groups (6 months: 6.5 vs $6.6 \mathrm{~kg}, p=0.71 ; 2$ years: 3.6 vs $4.5 \mathrm{~kg}, p=0.11)$ and between the low- and high-fat diet groups ( 6 months: 6.7 vs $6.4 \mathrm{~kg}, p=0.52 ; 2$ years: $4.1 \mathrm{vs} 3.9 \mathrm{~kg}, p=0.76$ ) among study participants (ESM Table 2). The reported dietary intakes confirmed that participants modified their intake of macronutrients in the direction of the intervention (ESM Table 3). Participants in the high-protein diet groups and in the highfat diet groups reported higher dietary protein intake and higher fat intake than those in the low-protein diet groups and in the low-fat diet groups, respectively (all $p<0.05$ ).

Genotypes and changes in weight, glucose and insulin resistance by diet groups We observed significant interactions between DHCR7 rs12785878 and high-/low-protein diet intervention on changes in fasting insulin $(p$ for interaction $=0.0008)$ and HOMA-IR ( $p$ for interaction=0.0009) at 6 months, adjusted for age, sex, ethnicity and the baseline value for the respective outcome trait (Table 2). In the high-protein diet groups, the T-allele of DHCR7 rs12785878 was associated with greater decreases in fasting insulin $(p=0.0009)$ and HOMA-IR ( $p=0.002$; Table 2 and Fig. 1$)$. These genotype-diet interactions and genotype effects remained significant after correcting for multiple testing $(p<0.0028)$. In the low-protein diet groups, there was no significant genotype effect on changes in fasting insulin or HOMA-IR ( $p \geq 0.14$ ). Results were similar but attenuated at 2 years (Table 2 and Fig. 1), and there were nominally significant genotype-diet interactions on changes in fasting 


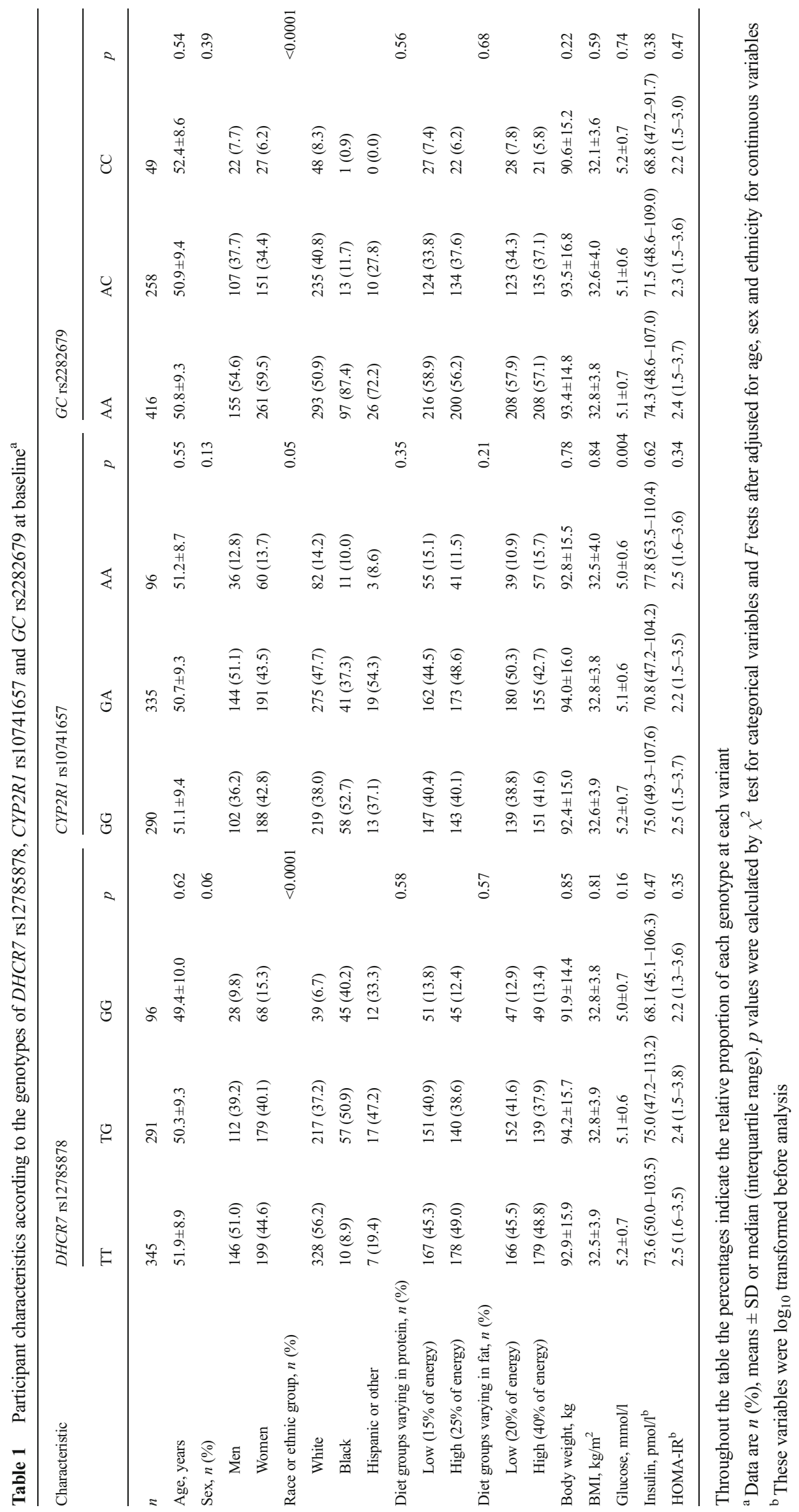


Table 2 Effects of DHCR7 rs12785878, CYP2R1 rs10741657 and GC rs2282679 on changes in body weight, glucose, insulin and HOMA-IR in response to low-/high-protein $\operatorname{diets}^{\mathrm{a}}$

\begin{tabular}{|c|c|c|c|c|c|c|c|c|c|c|c|c|}
\hline \multirow[t]{3}{*}{ Characteristic } & \multicolumn{4}{|c|}{ DHCR7 rs 12785878} & \multicolumn{4}{|c|}{ CYP2R1 rs10741657 } & \multicolumn{4}{|c|}{$G C \operatorname{rs} 2282679$} \\
\hline & \multicolumn{2}{|l|}{6 months } & \multicolumn{2}{|l|}{2 years } & \multicolumn{2}{|l|}{6 months } & \multicolumn{2}{|l|}{2 years } & \multicolumn{2}{|l|}{6 months } & \multicolumn{2}{|l|}{2 years } \\
\hline & $\beta \pm \mathrm{SE}$ & $p$ & $\beta \pm \mathrm{SE}$ & $p$ & $\beta \pm \mathrm{SE}$ & $p$ & $\beta \pm \mathrm{SE}$ & $p$ & $\beta \pm \mathrm{SE}$ & $p$ & $\beta \pm \mathrm{SE}$ & $p$ \\
\hline \multicolumn{13}{|c|}{ Change in body weight, $\mathrm{kg}$} \\
\hline Low-protein diet & $-0.62 \pm 0.51$ & 0.22 & $-0.50 \pm 0.69$ & 0.46 & $-0.33 \pm 0.45$ & 0.46 & $-0.43 \pm 0.64$ & 0.50 & $0.89 \pm 0.52$ & 0.08 & $1.05 \pm 0.73$ & 0.15 \\
\hline High-protein diet & $-1.18 \pm 0.47$ & 0.01 & $-0.96 \pm 0.66$ & 0.14 & $0.19 \pm 0.43$ & 0.66 & $0.73 \pm 0.58$ & 0.21 & $0.40 \pm 0.48$ & 0.41 & $0.19 \pm 0.65$ & 0.78 \\
\hline$p$ for interaction & & 0.25 & & 0.52 & & 0.48 & & 0.19 & & 0.68 & & 0.41 \\
\hline \multicolumn{13}{|c|}{ Change in glucose, $\mathrm{mmol} / \mathrm{l}$} \\
\hline Low-protein diet & $0.05 \pm 0.04$ & 0.23 & $0.08 \pm 0.05$ & 0.08 & $-0.02 \pm 0.04$ & 0.57 & $0.01 \pm 0.04$ & 0.91 & $0.01 \pm 0.04$ & 0.84 & $0.01 \pm 0.05$ & 0.94 \\
\hline High-protein diet & $-0.02 \pm 0.04$ & 0.68 & $-0.05 \pm 0.05$ & 0.35 & $-0.03 \pm 0.04$ & 0.42 & $0.04 \pm 0.05$ & 0.44 & $-0.01 \pm 0.04$ & 0.95 & $-0.01 \pm 0.05$ & 0.95 \\
\hline$p$ for interaction & & 0.30 & & 0.07 & & 0.95 & & 0.71 & & 0.82 & & 0.93 \\
\hline \multicolumn{13}{|c|}{ Change in log insulin, pmol/1 } \\
\hline Low-protein diet & $0.05 \pm 0.04$ & 0.19 & $0.05 \pm 0.04$ & 0.25 & $0.04 \pm 0.04$ & 0.32 & $0.004 \pm 0.04$ & 0.92 & $0.05 \pm 0.04$ & 0.24 & $-0.01 \pm 0.05$ & 0.80 \\
\hline High-protein diet & $-0.13 \pm 0.04$ & 0.0009 & $-0.06 \pm 0.04$ & 0.15 & $0.02 \pm 0.04$ & 0.56 & $0.04 \pm 0.04$ & 0.34 & $0.04 \pm 0.04$ & 0.29 & $0.01 \pm 0.04$ & 0.72 \\
\hline$p$ for interaction & & 0.0008 & & 0.03 & & 0.74 & & 0.63 & & 0.91 & & 0.54 \\
\hline \multicolumn{13}{|l|}{ Change in log HOMA-IR } \\
\hline Low-protein diet & $0.06 \pm 0.04$ & 0.14 & $0.06 \pm 0.05$ & 0.19 & $0.03 \pm 0.04$ & 0.47 & $0.005 \pm 0.05$ & 0.92 & $0.05 \pm 0.04$ & 0.25 & $-0.02 \pm 0.05$ & 0.76 \\
\hline High-protein diet & $-0.13 \pm 0.04$ & 0.002 & $-0.06 \pm 0.05$ & 0.16 & $0.02 \pm 0.04$ & 0.70 & $0.04 \pm 0.04$ & 0.30 & $0.04 \pm 0.04$ & 0.35 & $0.02 \pm 0.05$ & 0.74 \\
\hline$p$ for interaction & & 0.0009 & & 0.02 & & 0.8 & & 0.57 & & 0.99 & & 0.51 \\
\hline
\end{tabular}

${ }^{\mathrm{a}}$ Data are $\beta \mathrm{s} \pm \mathrm{SE}$, representing effect size for each vitamin-D-increasing allele of $D H C R 7 \mathrm{rs} 12785878$ (T allele), CYP2R1 rs10741657 (A allele) and GC rs2282679 (A allele), respectively, on change in each trait, adjusted for age, sex, ethnicity and the baseline value for the respective outcome trait

insulin ( $p$ for interaction $=0.03$ ) and HOMA-IR ( $p$ for interaction $=0.02$ ). These results did not change materially after further adjustment for weight loss (ESM Table 4).

For genetic variants in $C Y P 2 R 1$ and $G C$, we did not find significant genotype-diet interactions or genotype effects on changes in body weight, fasting glucose, fasting insulin or HOMA-IR at 6 months or 2 years (all $p>0.05$; Table 2).

We also examined interaction between genetic variants and high-/low-fat diet interventions on changes in these metabolic traits (ESM Table 5). There were nominally significant interactions between CYP2R1 rs10741657 and dietary fat on changes in fasting insulin and HOMA-IR at both 6 months and 2 years ( $\mathrm{p}$ for interaction $<0.05$ ). In the low-fat diet groups, the A allele of CYP2R1 rs10741657 showed a trend to be associated with less decreases in fasting insulin and HOMA-IR at both 6 months $(p=0.04)$ and 2 years $(p=0.03)$, while no genotype effect was observed in the high-fat diet groups. However, these results did not pass a threshold of $p<0.0028$ after correcting for multiple testing.

As the majority of participants in this study were white ( $\sim 80 \%)$, we further performed sensitivity analyses by excluding other ethnic groups. Similar results were observed when the analyses were restricted to the white participants.
Trajectory of changes in insulin resistance by $D H C R 7$ rs12785878 in response to high-/low-protein diets We then assessed the genotype of DHCR7 rs 12785878 by time effect on improvement of insulin resistance over the 2 year intervention in the participants assigned to the high- and low-protein diets (Fig. 2). In the high-protein diet group, participants carrying the T-allele of DHCR7 rs12785878 (TT and TG genotypes) had a greater improvement of fasting insulin $(p<0.0001)$ and HOMA-IR $(P=0.0009)$ than non-T-carriers (GG genotype) across the 2 year intervention. As expected, the genetic associations with changes in fasting insulin and HOMA-IR appeared to be more pronounced at 6 months than at 2 years. In the low-protein diet group, there was no significant genotype effect on the trajectory of changes in insulin resistance over the 2 year intervention $(p \geq 0.11)$. Similar results were observed when the analyses were restricted to the white participants.

\section{Discussion}

In this large, 2 year randomised dietary weight-loss intervention trial, we found significant interactions between a vitaminD-related genetic variant $D H C R 7$ rs 12785878 and dietary protein intake on changes in fasting insulin and HOMA-IR; 

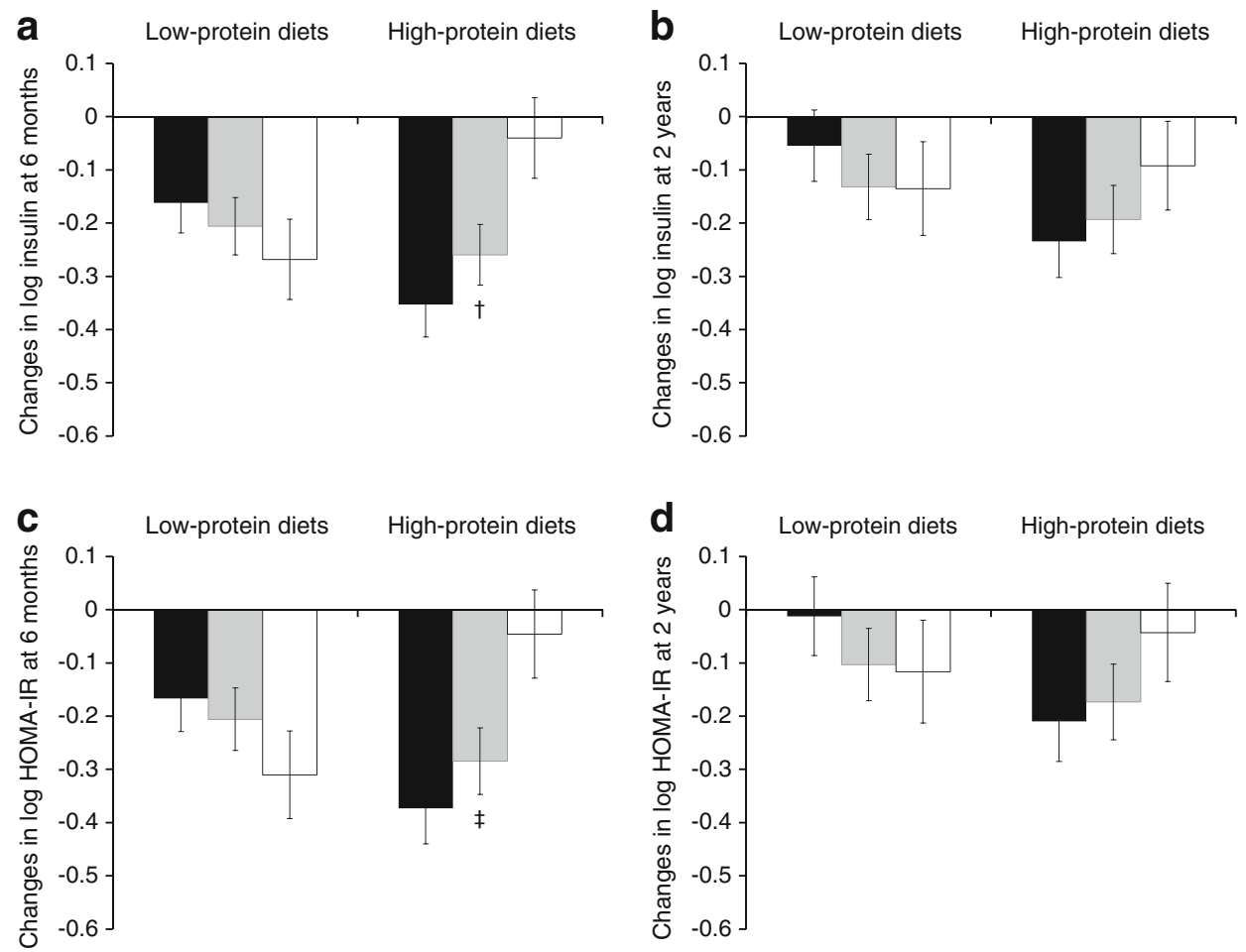

Fig. 1 Changes in insulin (a, b) and HOMA-IR (c, d) by DHCR7 rs 12785878 genotype in response to low-protein diets and high-protein diets at 6 months and 2 years. Black bars, DHCR7 rs12785878 TT genotype; grey bars, DHCR7 rs12785878 TG genotype; white bars, DHCR7 rs12785878 GG genotype. Data are means $\pm \mathrm{SE}$, adjusted for age, sex, ethnicity and baseline value for the respective outcome traits. $p=0.0008$ and 0.03 for interactions between $D H C R 7$ rs 12785878 and diet

intervention on changes in fasting insulin at 6 months (a) and 2 years (b), respectively. $p=0.0009$ and 0.02 for interactions between $D H C R 7$ rs 12785878 and diet intervention on changes in HOMA-IR at 6 months (c) and 2 years (d), respectively. ${ }^{\dagger} p=0.0009$ and ${ }^{\star} p=0.002$ for genotype effects on changes in insulin (a) and HOMA-IR (c), respectively, at 6 months in the high-protein diet groups

Fig. 2 Trajectory of changes in insulin and HOMA-IR by DHCR7 rs 12785878 genotype in response to low-protein diets (a, c) and high-protein diets (b, d) over a 2 year intervention. Black circle and solid line, $D H C R 7$ rs12785878 TT genotype; grey circle and dashed line, DHCR7 rs12785878 TG genotype; white circle and grey dotted line, DHCR7 rs12785878 GG

genotype. Data are means $\pm \mathrm{SE}$, adjusted for age, sex, ethnicity and baseline value for the respective outcome traits. ${ }^{\dagger} p<0.0001$ and ${ }^{\star} p=0.0009$ for genotype effects on changes in insulin (b) and HOMA-IR (d), respectively, in the high-protein diet groups a
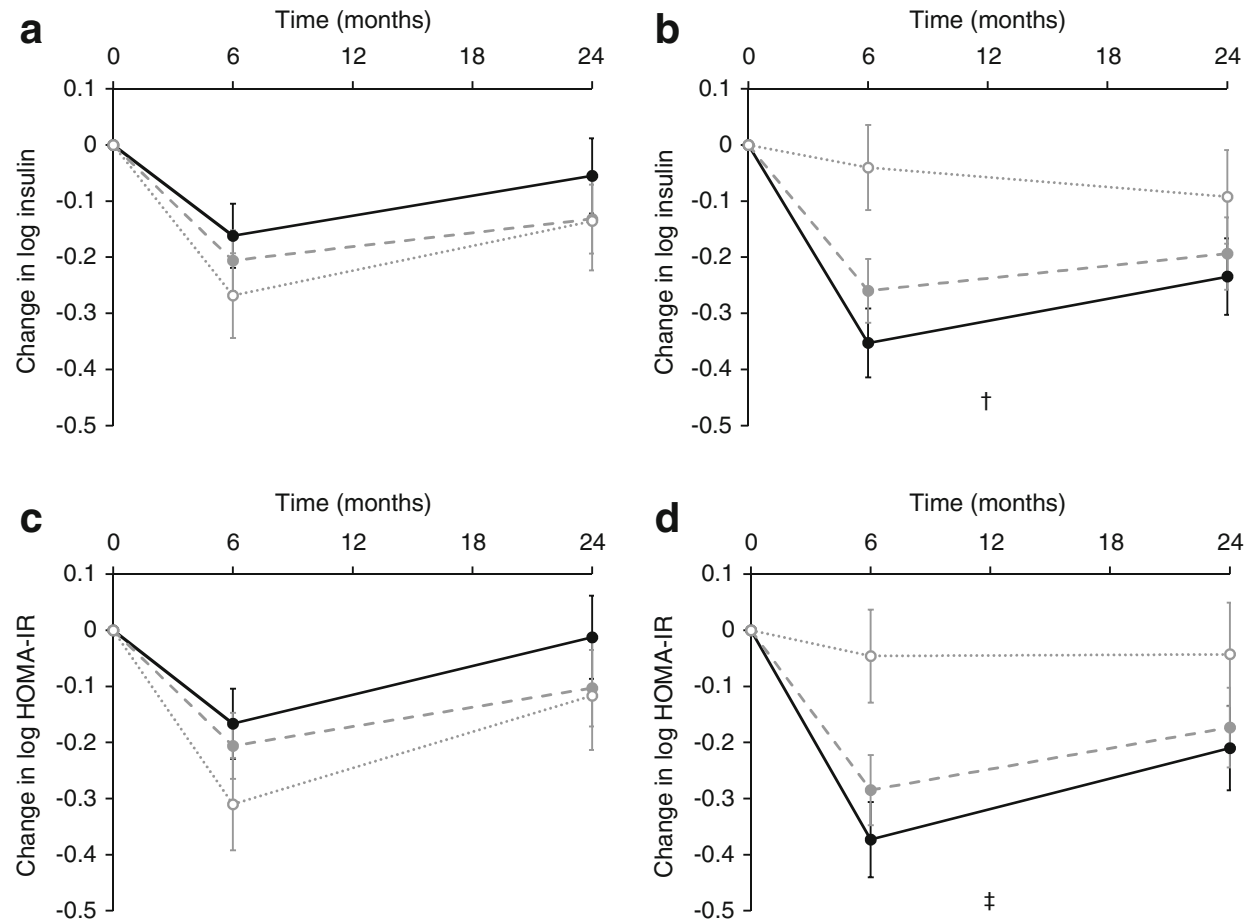

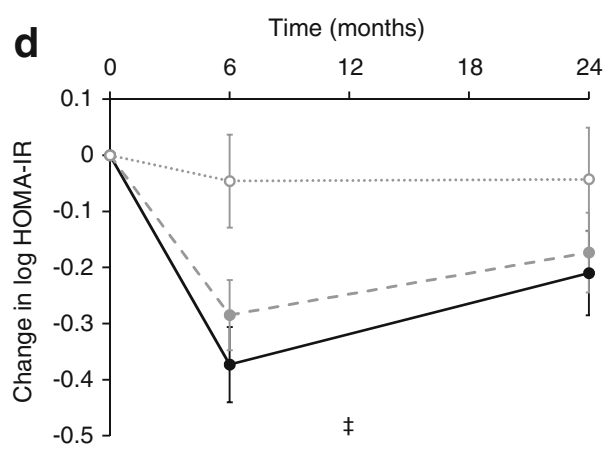


furthermore, results were consistent for short-term (6 months) and long-term ( 2 years) changes. The T-allele of DHCR7 rs 12785878 was associated with greater improvement of insulin resistance among individuals who were assigned to the high-protein diets.

A large body of evidence implicates the DHCR7 gene in insulin resistance through effects on vitamin D levels. The T allele of DHCR7 rs12785878 was strongly associated with higher circulating $25(\mathrm{OH}) \mathrm{D}$ levels in GWAS $\left(p=2.1 \times 10^{-27}\right)$ [9], while lower $25(\mathrm{OH}) \mathrm{D}$ levels have been associated with obesity, insulin resistance, systemic inflammation and the risk of diabetes [2-8]. A recent Mendelian randomisation study found that the $D H C R 7$ allele score but not the $C Y P 2 R 1$ allele score was associated with the risk of type 2 diabetes [14]. Several indices of insulin resistance were found to be significantly different across DHCR7 rs12785878 genotypes in women with polycystic ovary syndrome [23]. In addition, DHCR7 genetic variation was recently reported to interact with type 2 diabetes status to influence subclinical atherosclerosis [24]. However, no studies have investigated the interactions between $D H C R 7$ genetic variants and diet/lifestyle factors in relation to cardiometabolic traits.

Although the mechanism underlying the observed DHCR7 rs12785878 gene-dietary protein interaction is unclear, several potential biological processes might be implicated. A perfect proxy SNP of DHCR7 rs $12785878\left(r^{2}=1\right)$ has been found to modulate $D H C R 7$ mRNA levels in liver [24]. In addition, protein-rich foods (e.g. fish, dairy if fortified and meat to a lesser extent) are the main sources of naturally occurring cholecalciferol (vitamin $\mathrm{D}_{3}$ ) [20], and cholecalciferol could suppress DHCR7 activity in human skin cells [19]. In POUNDS Lost, the difference between high- and low-protein diets is mainly from the difference in consumption of skinless turkey, lean beef and skimmed/nonfat milk, while the difference between high- and low-fat diets is mainly from the difference in consumption of walnuts and olive oil [21]. Thus, there might be a greater difference in vitamin $\mathrm{D}$ content between high- and low-protein diets than that between high- and lowfat diets. However, we could not exclude the possibility that other dietary nutrients might be also involved, since it has been suggested that $D H C R 7$ gene expression might be regulated by dietary alpha-tocopherol [16], cholestyramine-plusLovastatin diet [17] and soy protein [18] in rats. In addition, dietary protein and amino acids are important modulators of glucose metabolism and insulin resistance [25-27]. Taken together, we speculate that cholecalciferol and/or other nutrients from protein-rich foods may interact with DHCR7 rs12785878 (or a causal variant indicated by this SNP) to influence DHCR7 function, thereby affecting vitamin D levels and eventually modifying the effects on changes in insulin resistance.

Diminished adherence occurred between 6 months and 2 years in POUND Lost [21]; therefore, it was not surprising that the genetic effects on changes in insulin resistance were attenuated at 2 years. Improvement of insulin resistance has been associated with changes in body weight in this intervention study. However, we did not find significant gene-diet interaction or genetic effects on changes in body weight, although it has been previously suggested that adiposity might be involved in the relationships between vitamin-D-related variants, 25(OH)D levels and type 2 diabetes [14]. Moreover, the genetic effects on improvement of insulin resistance were independent of weight loss, suggesting other pathways might be involved.

We did not find strong evidence for interactions between the other two vitamin-D-related genetic variants, CYP2R1 rs10741657 and $G C$ rs2282679, and dietary interventions on changes in body weight or glucose/insulin metabolism in this 2 year weight-loss trial. Both genes have been largely implicated in vitamin D metabolism, and CYP2R1 rs10741657 $\left(p=3.3 \times 10^{-20}\right)$ and $G C$ rs2282679 $\left(p=1.9 \times 10^{-109}\right)$ were also strongly associated with circulating $25(\mathrm{OH}) \mathrm{D}$ levels in GWAS [9]. However, a recent study found that the expression levels of CYP2R1 in adipose tissues did not change during dietinduced weight loss [28]. Nevertheless, further studies are warranted to validate the observed nominally significant interactions between CYP2R1 rs10741657 and dietary fat on changes in fasting insulin and HOMA-IR. It has been suggested that the quality of dietary fat influences glucose metabolism and insulin resistance [29]. However, it should be noted that diets varying in fat content (low-fat diets or high-fat diets) are not emphasised in the nutritional therapy recommendations for diabetes [30], and both extremely high- and low-fat diets might not be beneficial regarding glucose metabolism in diabetes [29].

To the best of our knowledge, this is the first study to show significant interactions between vitamin-D-related genetic variation and dietary protein on improvement of insulin resistance in a large and long-term randomised trial. Previous evidence has suggested the important implications of vitamin $\mathrm{D}$ in insulin resistance and type 2 diabetes. Our findings provide new insight into the utility of using knowledge of vitamin-D-related genetic variation to improve personalised dietary interventions. However, several limitations of this study should be addressed. We did not measure circulating vitamin $\mathrm{D}$ or $25(\mathrm{OH}) \mathrm{D}$ concentrations in this study, which limited our analysis to examine the role of vitamin D levels in the gene-diet interaction and explore potential underlying mechanisms. Although our major findings remained significant after adjustment for multiple tests, replication of these results is needed in future. In addition, the majority of participants are white $(80 \%)$ in our study and thus further studies are needed in other ethnic groups, since significant ethnic differences in circulating vitamin D levels and related genetic variants have been acknowledged [31]. 
In summary, our data indicate that dietary protein may modify the effect of the $D H C R 7$ rs 12785878 variant on changes in insulin resistance over a 2 year dietary intervention. Individuals carrying the $\mathrm{T}$ allele (vitamin-D-increasing allele) of DHCR7 rs 12785878 might benefit more in improvement of insulin resistance than noncarriers by consuming high-protein weight-loss diets. Our findings of gene-protein dietary interaction are biologically plausible, provide additional evidence for the roles of vitamin $\mathrm{D}$ in insulin resistance, and suggest new insights into effective strategies for type 2 diabetes prevention and intervention.

Acknowledgements We are particularly grateful to all participants in the trial for their dedication and contribution to the research.

Funding This study was supported by grants from the National Heart, Lung, and Blood Institute (HL071981), the Boston Obesity Nutrition Research Center (P30 DK46200) and the National Institute of Diabetes and Digestive and Kidney Diseases (DK091718). QQ is a recipient of a Scientist Development Award (K01HL129892) from the National Heart, Lung, and Blood Institute of the National Institutes of Health.

Duality of interest The authors declare that there is no duality of interest associated with this manuscript.

Contribution statement $\mathrm{QQ}$ and $\mathrm{YZ}$ contributed to conception and design, performed the statistical analyses and drafted the manuscript. $\mathrm{TH}$ and JR contributed to analysis and interpretation of data, and manuscript revision. GAB, FMS and LQ contributed to conception and design, acquisition and interpretation of the data, and manuscript revision. All authors contributed to critical revisions and have read and approved the final manuscript. LQ is the guarantor of this work

\section{References}

1. Nagpal S, Na S, Rathnachalam R (2005) Noncalcemic actions of vitamin D receptor ligands. Endocr Rev 26:662-687

2. Mitri J, Pittas AG (2014) Vitamin D and diabetes. Endocrinol Metab Clin North Am 43:205-232

3. Song Y, Wang L, Pittas AG et al (2013) Blood 25-hydroxy vitamin D levels and incident type 2 diabetes: a meta-analysis of prospective studies. Diabetes Care 36:1422-1428

4. Vimaleswaran KS, Berry DJ, Lu C et al (2013) Causal relationship between obesity and vitamin D status: bi-directional Mendelian randomization analysis of multiple cohorts. PLoS Med 10: e1001383

5. Guadarrama-Lopez AL, Valdes-Ramos R, Martinez-Carrillo BE (2014) Type 2 diabetes, PUFAs, and vitamin D: their relation to inflammation. J Immunol Res 2014:860703

6. Muscogiuri G, Sorice GP, Prioletta A et al (2010) 25Hydroxyvitamin D concentration correlates with insulinsensitivity and BMI in obesity. Obesity (Silver Spring) 18:19061910

7. Alemzadeh R, Kichler J, Babar G, Calhoun M (2008) Hypovitaminosis D in obese children and adolescents: relationship with adiposity, insulin sensitivity, ethnicity, and season. Metabolism 57:183-191

8. Lu L, Yu Z, Pan A et al (2009) Plasma 25-hydroxyvitamin D concentration and metabolic syndrome among middle-aged and elderly Chinese individuals. Diabetes Care 32:1278-1283
9. Wang TJ, Zhang F, Richards JB et al (2010) Common genetic determinants of vitamin $\mathrm{D}$ insufficiency: a genome-wide association study. Lancet 376:180-188

10. Kuan V, Martineau AR, Griffiths CJ, Hypponen E, Walton R (2013) DHCR7 mutations linked to higher vitamin D status allowed early human migration to northern latitudes. BMC Evol Biol 13:144

11. Cheng JB, Levine MA, Bell NH, Mangelsdorf DJ, Russell DW (2004) Genetic evidence that the human CYP2R1 enzyme is a key vitamin D 25-hydroxylase. Proc Natl Acad Sci U S A 101: 7711-7715

12. Nagasawa H, Uto Y, Sasaki H et al (2005) Gc protein (vitamin Dbinding protein): Gc genotyping and GcMAF precursor activity. Anticancer Res 25:3689-3695

13. Vimaleswaran KS, Cavadino A, Berry DJ et al (2014) Association of vitamin D status with arterial blood pressure and hypertension risk: a Mendelian randomisation study. Lancet Diabetes Endocrinol 2:719-729

14. Afzal S, Brondum-Jacobsen P, Bojesen SE, Nordestgaard BG (2014) Vitamin D concentration, obesity, and risk of diabetes: a Mendelian randomisation study. Lancet Diabetes Endocrinol 2: 298-306

15. Ye Z, Sharp SJ, Burgess S et al (2015) Association between circulating 25-hydroxyvitamin $\mathrm{D}$ and incident type 2 diabetes: a Mendelian randomisation study. Lancet Diabetes Endocrinol 3: $35-42$

16. Rota C, Barella L, Minihane AM, Stocklin E, Rimbach G (2004) Dietary alpha-tocopherol affects differential gene expression in rat testes. IUBMB Life 56:277-280

17. Kim JH, Lee JN, Paik YK (2001) Cholesterol biosynthesis from lanosterol. A concerted role for Sp1 and NF-Y-binding sites for sterol-mediated regulation of rat 7-dehydrocholesterol reductase gene expression. J Biol Chem 276:18153-18160

18. Su Y, Shankar K, Simmen RC (2009) Early soy exposure via maternal diet regulates rat mammary epithelial differentiation by paracrine signaling from stromal adipocytes. J Nutr 139:945-951

19. Zou L, Porter TD (2015) Rapid suppression of 7dehydrocholesterol reductase activity in keratinocytes by vitamin D. J Steroid Biochem Mol Biol 148:64-71

20. Schmid A, Walther B (2013) Natural vitamin D content in animal products. Adv Nutr 4:453-462

21. Sacks FM, Bray GA, Carey VJ et al (2009) Comparison of weightloss diets with different compositions of fat, protein, and carbohydrates. N Engl J Med 360:859-873

22. Matthews DR, Hosker JP, Rudenski AS, Naylor BA, Treacher DF, Turner RC (1985) Homeostasis model assessment: insulin resistance and beta-cell function from fasting plasma glucose and insulin concentrations in man. Diabetologia 28:412-419

23. Wehr E, Trummer O, Giuliani A, Gruber HJ, Pieber TR, Obermayer-Pietsch B (2011) Vitamin D-associated polymorphisms are related to insulin resistance and vitamin D deficiency in polycystic ovary syndrome. Eur J Endocrinol 164:741-749

24. Strawbridge RJ, Deleskog A, McLeod O et al (2014) A serum 25hydroxyvitamin D concentration-associated genetic variant in DHCR7 interacts with type 2 diabetes status to influence subclinical atherosclerosis (measured by carotid intima-media thickness). Diabetologia 57:1159-1172

25. Tremblay F, Lavigne C, Jacques H, Marette A (2007) Role of dietary proteins and amino acids in the pathogenesis of insulin resistance. Annu Rev Nutr 27:293-310

26. Wang TJ, Larson MG, Vasan RS et al (2011) Metabolite profiles and the risk of developing diabetes. Nat Med 17:448-453

27. Cheng S, Rhee EP, Larson MG et al (2012) Metabolite profiling identifies pathways associated with metabolic risk in humans. Circulation 125:2222-2231

28. Wamberg L, Christiansen T, Paulsen SK et al (2013) Expression of vitamin D-metabolizing enzymes in human adipose tissue - the 
effect of obesity and diet-induced weight loss. Int J Obes (Lond) 37 : 651-657

29. Schwab U, Lauritzen L, Tholstrup T et al (2014) Effect of the amount and type of dietary fat on cardiometabolic risk factors and risk of developing type 2 diabetes, cardiovascular diseases, and cancer: a systematic review. Food Nutr Res 58:25145
30. Evert AB, Boucher JL, Cypress M et al (2014) Nutrition therapy recommendations for the management of adults with diabetes. Diabetes Care 37(Suppl 1):S120-S143

31. Powe CE, Evans MK, Wenger J et al (2013) Vitamin D-binding protein and vitamin D status of black Americans and white Americans. N Engl J Med 369:1991-2000 